\title{
Evolving Concepts in the Diagnosis and Staging of Multiple Myeloma
}

Presented by Jens Hillengass, MD

\section{ABSTRACT}

Until recently, treatment for multiple myeloma was only initiated in symptomatic disease. Greater understanding of the underlying biology and the development of new biomarkers, however, has led to changes in diagnosis, and with the help of improved imaging techniques, clinicians are increasingly identifying patients with early myeloma who may benefit from treatment. One major development in diagnostic imaging is the replacement of radiography with CT. PET/CT imaging is also useful for treatment response assessment, because the disappearance of focal lesions is an important prognostic factor. Finally, the Revised International Staging System for multiple myeloma has incorporated additional prognostic markers of aggressive disease, and risk-adapted treatment has been shown to improve survival.

J Natl Compr Canc Netw 2020;18(12.5):1770-1772 doi: 10.6004/jnccn.2020.5041

Despite significant developments in diagnosis and treatment, multiple myeloma (MM) remains incurable in most patients, and median survival is only 6 to 8 years. As patients continue to live longer with this disease, however, quality of life becomes increasingly important, according to Jens Hillengass, MD, Chief of Myeloma, Roswell Park Comprehensive Cancer Center, during the NCCN 2020 Virtual Congress: Hematologic Malignancies. "Bone destruction, kidney failure, and anemia caused by replacement of physiologic hematopoiesis have a significant impact on quality of life," said Dr. Hillengass. "This raises the question of whether earlier treatment would improve the prognosis of those patients."

At the congress, Dr. Hillengass described the updated diagnostic criteria by the International Myeloma Working Group, listed imaging techniques that are critical for accurate diagnosis, and reviewed the rationale for development of the Revised International Staging System (R-ISS) for MM.

\section{New Diagnostic Criteria}

As Dr. Hillengass explained, the pathophysiology of MM is characterized by 4 components: replacement of physiologic hematopoiesis, bone destruction, hypercalcemia, and renal insufficiency. Although the most advanced stage of the disease is MM itself, some patients are diagnosed earlier with monoclonal gammopathy of undetermined significance (MGUS) or smoldering MM.

The clinical definition of MGUS is $<10 \%$ clonal plasma cells in the bone marrow, lower monoclonal protein levels in serum and/or urine $(<30 \mathrm{~g} / \mathrm{L})$, and no end organ damage. For smoldering $\mathrm{MM}$, patients must have $>10 \%$ clonal plasma cells, higher levels of monoclonal protein in serum and/or urine ( $30 \mathrm{~g} / \mathrm{L}$ ), and no end organ damage.

Although MM used to be defined as the presence of end organ damage, this has been changed to myelomadefining events to identify patients who may be at risk of developing symptoms.

"It was very important to develop biomarkers to identify those patients with early myeloma who would benefit from treatment versus those have very low risk of progressing in the future," said Dr. Hillengass, who noted that patients with MGUS have a progression risk of approximately $1 \%$ per year. "While some patients with smoldering MM have a similarly low risk (as MGUS), others have a very high risk of progression."

Another reason to treat early, but not too early, is that myeloma can evolve genetically over the course of the disease. There are also influences on the microenvironment, so it is important not to wait until the disease progresses with symptoms, said Dr. Hillengass.

\section{High-Risk Smoldering MM}

As Dr. Hillengass reported, several factors have been identified to distinguish high-risk smoldering MM. ${ }^{2}$ Diagnostics may involve bone marrow plasma cells, monoclonal protein, serum free light chains, flow cytometry, immunoparesis, MRI, and fluorescence in situ hybridization (FISH).

Clinicians should also consider the concept of evolving smoldering MM, said Dr. Hillengass, because 
dynamically stable markers of disease are associated with lower risk of progression versus markers that are increasing.

As Dr. Hillengass reported, 3 new biomarkers constitute myeloma-defining events. First, the presence of more than one focal lesion on spine or whole-body MRI is associated with a higher risk of progression to symptomatic MM. ${ }^{3}$ A markedly elevated serum free light chain ratio $(\geq 100)$ and involved free light chain levels $\geq 100 \mathrm{mg} / \mathrm{L}$ are also markers of malignant transformation. ${ }^{4}$ Finally, said Dr. Hillengass, the strongest new biomarker to identify high-risk smoldering $\mathrm{MM}$ is the percentage of monoclonal plasma cells in the bone marrow, with $\geq 60 \%$ being prognostic of disease progression. $^{5}$

Those myeloma-defining events have been added to the standard CRAB (calcium elevation, renal failure, anemia, lytic bone lesions) criteria ${ }^{6}$ :

- Hypercalcemia: serum calcium $>0.25 \mathrm{mmol} / \mathrm{L}$ above upper limit of normal or $>2.75 \mathrm{mmol} / \mathrm{L}$ ( $>1 \mathrm{mg} / \mathrm{dL}$ above upper limit of normal);

- Renal insufficiency: creatinine clearance $<40 \mathrm{~mL} /$ min or serum creatinine $>173 \mu \mathrm{mol} / \mathrm{L}(>2 \mathrm{mg} / \mathrm{dL}$ );

- Anemia: normochromic, normocytic with a hemoglobin value of $>2 \mathrm{~g} / \mathrm{dL}$ below the lower limit of normal, or a hemoglobin value $<10 \mathrm{~g} / \mathrm{dL}$; and

- Bone lesions: lytic lesions detected by radiography, CT, or PET/CT.

\section{Imaging Techniques}

With respect to imaging techniques in MM, Dr. Hillengass differentiated bone imaging from bone marrow imaging. Bone imaging reveals the result of MM-the overactivation of osteoclasts-and bone marrow imaging shows what is going in the bone marrow, where the actual disease takes place.

According to Dr. Hillengass, bone imaging is important for diagnosis, because osteolytic lesions are a reason to treat. However, it is also used for localization of pain and prevention of complications. Bone marrow imaging, conversely, is used to identify focal lesions, which can indicate risk of progression. Even if they have not caused bone destruction, said Dr. Hillengass, focal lesions constitute a strong prognostic marker that requires treatment. Clinicians can also localize pain and prevent complications based on imaging of the bone marrow. Finally, bone marrow imaging is playing an increasingly important role in response assessment.

One major development in imaging in $\mathrm{MM}$ is the replacement of radiography with CT. A study of the International Myeloma Working Group found that $25 \%$ of patients with a negative radiograph had a positive CT for osteolytic lesions. ${ }^{7}$

MRI shows different infiltration patterns: minimal, diffuse, focal, or mixed between the focal and diffuse infiltration. Knowing about these different patterns is very important because a diagnosis with a random bone marrow sample of the pelvis, for example, might otherwise be missed, said Dr. Hillengass.

MRI is also helpful in patients who have osteoporosis. Although osteoporosis is no longer identified as a symptom of MM in the new guidelines, some patients may have osteoporotic-looking bones, said Dr. Hillengass, and MRI can be used to differentiate bone marrow cellularity and distinguish between benign versus malignant fractures.

Finally, PET/CT imaging is useful for treatment response assessment, because multiple studies have shown that the disappearance of focal lesions is an important prognostic factor. Thus, even in patients with complete remission, the presence of focal lesions is an adverse prognostic factor, said Dr. Hillengass. When combined with minimal residual disease (MRD) negativity, patients with a deep remission after treatment and PET negativity have a very good prognosis.

\section{Risk Stratification}

According to Dr. Hillengass, risk stratification in $\mathrm{MM}$ is becoming increasingly important. The most widely applied risk stratification system is the ISS, which uses 2 serum markers: beta- 2 microglobulin and albumin. Low beta-2 microglobulin and high albumin levels are associated with a good prognosis, whereas high beta-2 microglobulin and low albumin levels are associated with an adverse prognosis.

The R-ISS has added FISH cytogenetics [deletion of $17 p$, translocation $t(4 ; 14)$ and translocation $t(14 ; 16)]$ and lactate dehydrogenase as prognostic markers of aggressive disease. ${ }^{8}$ The former can be used to guide treatment, said Dr. Hillengass, with bortezomib-based regimens outperforming thalidomide-based treatment in patients with high-risk FISH cytogenetics in a historic study. ${ }^{9}$ Other markers associated with an adverse prognosis have also recently been published: gain (1q) and deletion (1p).

Risk-adapted treatment is not only about high-risk patients, said Dr. Hillengass. In certain patients with translocation $\mathrm{t}(11 ; 14)$, for example, venetoclax has been shown to work better. ${ }^{10}$

Another important prognostic factor is MRD negativity. Patients with standard risk who are negative for MRD have a very good prognosis. Patients with high-risk cytogenetics who are negative for MRD, however, have nearly the same risk. 
"It's still too early to adjust our treatment, but if a patient achieves MRD negativity, it's a very strong prognostic marker that they might overcome the relevance of high-risk cytogenetics in the future," said Dr. Hillengass.

The same is also true for ISS, he said. Patients with high ISS and MRD negativity still have a "pretty good prognosis" compared with those with high ISS and MRD positivity. "This is very relevant because we now have the tools to bring more patients into a deep MRD-negative stage," Dr. Hillengass concluded.

Disclosures: Dr. Hillengass has disclosed that he has received honoraria from Amgen and Janssen, has received grant/research support from Celgene, and is a scientific advisor for Adaptive, Amgen, Bristol-Myers Squibb Company, GlaxoSmithKline, Janssen, Oncopeptides, and Oncotracker.

Correspondence: Jens Hillengass, MD, Roswell Park Comprehensive Cancer Center, Elm and Carlton Streets, Buffalo, NY 14214

Email: jens.hillengass@roswellpark.org

\section{References}

1. Kyle RA, Remstein ED, Therneau TM, et al. Clinical course and prognosis of smoldering (asymptomatic) multiple myeloma. N Engl J Med 2007;356: 2582-2590.

2. Dispenzieri A, Stewart AK, Chanan-Khan A, et al. Smoldering multiple myeloma requiring treatment: time for a new definition? Blood 2013;122: 4172-4181.

3. Kastritis E, Moulopoulos LA, Terpos E, et al. The prognostic importance of the presence of more than one focal lesion in spine MRI of patients with asymptomatic (smoldering) multiple myeloma. Leukemia 2014;28: 2402-2403.

4. Larsen JT, Kumar SK, Dispenzieri A, et al. Serum free light chain ratio as a biomarker for high-risk smoldering multiple myeloma. Leukemia 2013;27: 941-946.

5. Rajkumar SV, Larson D, Kyle RA. Diagnosis of smoldering multiple myeloma. N Engl J Med 2011;365:474-475.
6. Rajkumar SV, Dimopoulos MA, Palumbo A, et al. International Myeloma Working Group updated criteria for the diagnosis of multiple myeloma. Lancet Oncol 2014;15:e538-548.

7. Hillengass J, Moulopoulos LA, Delorme S, et al. Whole-body computed tomography versus conventional skeletal survey in patients with multiple myeloma: a study of the International Myeloma Working Group [published online August 25, 2017]. Blood Cancer J, doi: 10.1038/bcj.2017.78

8. Palumbo A, Avet-Loiseau $\mathrm{H}$, Oliva $\mathrm{S}$, et al. Revised international staging system for multiple myeloma: a report from International Myeloma Working Group. J Clin Oncol 2015;33:2863-2869.

9. Sonneveld P, Avet-Loiseau H, Lonial S, et al. Treatment of multiple myeloma with high-risk cytogenetics: a consensus of the International Myeloma Working Group. Blood 2016;127:2955-2962.

10. Kumar S, Kaufman JL, Gasparetto C, et al. Efficacy of venetoclax as targeted therapy for relapsed/refractory $\mathrm{t}(11 ; 14)$ multiple myeloma. Blood 2017;130:2401-2409. 